\title{
Zur Bestimmung des Volumens biologischer Objekte ${ }^{1}$
}

\author{
HANSWERnER KeSSELER \\ Biologische Anstalt Helgoland, Meeresstation, Helgoland
}

ABSTRACT: On the volume determination of biological objects. A new simple glass-made apparatus for very exact volume determinations of biological material is described. The accuracy and reproducibility of the measurements are demonstrated.

\section{EINLEITUNG}

Für viele biologische Untersuchungen, insbesondere auf physiologischem Gebiet, ist die Kenntnis des Volumens lebender Organismen erforderlich. Wegen der besonderen Eigenschaften dieser Objekte lassen sich jedoch die üblichen physikalischen Bestimmungsmethoden (Pyknometer, archimedisches Prinzip; Näheres in KoHLrausch 1953, p. $49 \mathrm{ff}$.) nur in den seltensten Fällen mit befriedigendem Erfolg anwenden. Zudem sind diese indirekten Bestimmungsmethoden, bei denen das Volumen auf dem Umwege über mehrfache Wägungen unter verschiedenen Bedingungen (Luftgewicht, Tauchgewicht) und unter Berüicksichtigung der Temperatur des umgebenden Mediums ermittelt werden muß, meist sehr zeitraubend. Zur Umgehung dieser Schwierigkeiten begnügte man sich daher in den meisten Fällen damit, das Volumen des Untersuchungsobjektes durch Eintauchen in einen wassergefüllten Meßzylinder zu bestimmen. Diese Methode führt jedoch, besonders bei sehr sperrigem, aber wenig voluminösem Material beziehungsweise bei zarten Objekten mit großer relativer Oberfläche, zu sehr ungenauen Resultaten. MÜLLER-STOLL (1954) schlägt daher auf Grund seiner beim Arbeiten mit Ostseealgen gewonnenen Erfahrungen vor: „Bei der Dichteermittlung ist daher zweckmäßigerweise die direkte Volumenbestimmung zu umgehen..., da eine Volumenbestimmung auf Schwierigkeiten stößt" (p. 133). Die Bestimmung des Frischgewichtes, das dieser Autor zur Berechnung der Dichte seiner Algenthalli in Abwandlung des von KNIEP (1907) mitgeteilten Verfahrens benötigte, ist bei Wasserpflanzen jedoch kaum weniger problematisch.

Zur Vermeidung der genannten Nachteile aller herkömmlichen Bestimmungsmethoden wurde eine einfache Glasapparatur entwickelt, mit deren Hilfe direkte Volummessungen biologischer Objekte mit großer Genauigkeit ermöglicht werden. Zur Bestimmung des spezifischen Gewichtes ist außer der Volummessung nur e ine Wägung erforderlich.

1 Herrn Professor Dr. Adolf Bückmann zum 65. Geburtstag in Verehrung gewidmet. 


\section{BESCHREIBUNG DER APPARATUR}

Die in Abbildung 1 abgebildete Apparatur besteht aus einem als Meßgefäß dienenden Erlenmeyerkolben (1) mit Schliff, auf den vermittels eines Normschliff-Glashohlstopfens (2) Meßpipetten beziehungsweise -büretten (3) verschiedener Kaliber aufgesetzt werden können. An dem Kolben ist unten ein Eckhahn (4) angeschmolzen,

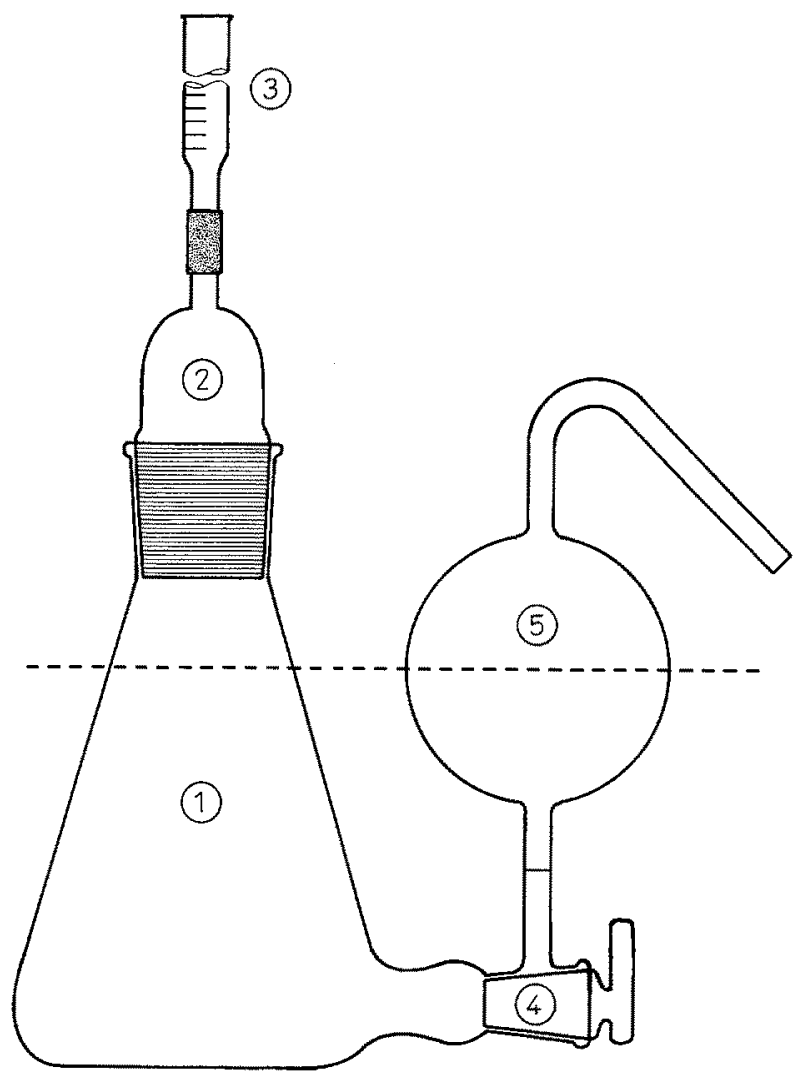

Abb. 1: Darstellung der Volumbestimmungsapparatur aus Glas (Erläuterungen im Text)

dessen Ablaufrohr in eine als Wasservorratsgefäß dienende Glashohlkugel (5) mündet. Das Verbindungsrohr ist mit einer Ringmarke versehen. Die Glaskugel steht über ein $z$ weites, gebogenes Rohr mit einem Gummi-Handgebläse (nicht eingezeichnet) in Verbindung.

\section{ARBEITSWEISE DES GERATES}

Die Ausführung einer Messung geschieht in folgender Weise: Zunächst werden Kolben und Kugel bei geöffnetem Hahn bis etwa in Höhe der gestrichelten Niveaulinie mit Wasser gefüllt. Sodann wird nach Aufsetzen einer geeigneten Meßbürette 
durch Betätigen des Gummigebläses das Wasser aus der Glaskugel in den Kolben gepumpt. Sobald der Wasserspiegel die Ringmarke ein wenig überschritten hat, wird der Hahn geschlossen. Die Wassermenge des Systems muß so bemessen sein, daß nach dem Überpumpen der Flïssigkeitsmeniskus in der Meßbürette in der Nähe des unteren Skalenendes liegt. Gegebenenfalls ist der Füllungszustand durch Nachfüllen oder Abgießen von Wasser zu korrigieren. Nach einer Wartezeit von etwa 1 Minute ist der Wassernachlauf von den Wänden der Glaskugel in das Verbindungsrohr zum Hahn beendet. Durch vorsichtiges Offnen des Hahnes wird nun der Wasserstand im Verbindungstohr genau auf die Ringmarke justiert. Der Meniskusstand in der Meßbïrette wird jetzt abgelesen und als Nullwert notiert.

Durch Offfnen des Hahnes läßt man nun das Wasser aus der Bürette wieder in den Kolben beziehungsweise in die Glaskugel zurückströmen, öffnet den Kolben und bringt das zu messende Objekt ein. Vorher überzeugt man sich davon - gegebenenfalls durch Eintauchen in einen Meßzylinder -, daß das Volumen des Meßobjektes das Fassungsvermögen der Bürette nicht übersteigt; andernfalls muß man vor der Messung eine entsprechende Wassermenge abpipettieren oder eine größere Bürette wählen. Die erstere Methode hat jedoch, zumal bei Verwendung geeichter Vollpipetten, den Vorzug größerer Genauigkeit. Die abpipettierte Wassermenge wird später zum Meßergebnis addiert. Nach dem Wiederaufsetzen der Bürette auf das Meßgefäß pumpt man erneut das Wasser aus der Vorratskugel in den Kolben und justiert in der beschriebenen Weise. Die Differenz zwischen erster und zweiter Bürettenablesung ergibt nach Addition der eventuell abpipettierten Wassermenge das gesuchte Volumen des Meßobjektes.

Die Genauigkeit einer solchen Messung läßt sich durch mehrfaches Wiederholen der Einstellungen noch wesentlich verbessern. Dabei ist vor jeder Einstellung die Bürette kurz abzunehmen, um Fehler, die durch geringfügiges Verkanten beziehungsweise unterschiedlich festes Andrücken des Glashohlstopfens entstehen können, zu eliminieren.

\section{GENAUIGKEIT DER METHODE}

Die Meßgenauigkeit der von mir benutzten Apparatur (300 ml Erlenmeyerkolben mit Normschliff NS 29/32 und 0,2 $\mathrm{ml} \mathrm{Meßbürette} \mathrm{mit} \mathrm{1/1000} \mathrm{ml} \mathrm{Skalenteilung)} \mathrm{und} \mathrm{die}$ Reproduzierbarkeit der damit erzielten Meßergebnisse sind aus Tabelle $1 \mathrm{zu}$ ersehen.

\section{Tabelle I}

Ergebnisse einer Serie von 12 Messungen; die Extremwerte (eingeklammert) wurden bei der Berechnung nicht berücksichtigt

\begin{tabular}{|cccc|}
\hline $\begin{array}{c}\text { Messung } \\
\text { Nr. }\end{array}$ & ermittelter Wert & $\begin{array}{c}\text { Messung } \\
\text { Nr. }\end{array}$ & ermittelter Wert \\
\hline 1 & $0,098 \mathrm{ml}$ & 7 & $0,099 \mathrm{ml}$ \\
2 & $0,084 \% "$ & 8 & $0,089, "$ \\
3 & $(0,031) "$ & 9 & $0,089, "$ \\
4 & $0,095 \%$ & 10 & $0,086, "$ \\
5 & $0,094 \%$ & 11 & $(0,058) "$ \\
6 & $0,083 \%$ & 12 & $0,099 "$, \\
\hline
\end{tabular}


Die Berechnung des Endergebnisses (E) dieser Meßreihe ergab

$$
\mathrm{E}=0,0916 \pm 0,002 \mathrm{ml}
$$

Dieses. Ergebnis besagt, daß das Volumen eines Objektes von $0,2 \mathrm{ml}$ Wasserverdrängung noch mit einer Genauigkeit von $1 \%$ bestimmt werden kann.

Diese Genauigkeit ist jedoch nur zu erzielen, wenn während der Messungen keine stärkeren Temperaturschwankungen im Arbeitsraum auftreten und die Apparatur nicht in der Nähe von starken Wärmestrahlern aufgestellt beziehungsweise dem direkten Sonnenlicht ausgesetzt wird. Die kubischen Ausdehnungskoeffizienten $(\alpha)$ von Jenaer Glas beziehungsweise von Wasser bei $18^{\circ} \mathrm{C}$ betragen nämlich nach KomLRausch (1953, Bd II, p. 547 f.):

$$
\begin{aligned}
& a_{\mathrm{J} . \mathrm{Gl} .} \approx 2,5 \cdot 10^{-5} \mathrm{grad}^{-1} \\
& \alpha_{\mathrm{H}_{2} \mathrm{O}}=19 \cdot 10^{-4} "
\end{aligned}
$$

Eine Temperaturänderung des wassergefüllten $(380 \mathrm{ml})$ Gerätes von $1^{\circ} \mathrm{C}$ würde demnach eine Volumänderung seines Inhalts von

$$
\Delta_{V}=0,065 \mathrm{ml}
$$

zur Folge haben.

Zur Erzielung einer möglichst großen Meßgenauigkeit muß daher auch jede Berührung der Apparatur, die einen Wärmeübergang zur Folge hat, vermieden werden. Das Gerät wird deshalb zweckmäßigerweise an einem Stativ befestigt, und der Glasstopfen nur unter Verwendung von Gummifingerlingen angefaßt. Unter Beachtung dieser Vorsichtsmaßnahmen erübrigt sich wegen der guten thermischen Isolationseigenschaften von Glas und der großen spezifischen Wärme des Wassers das Arbeiten in einem temperaturkonstanten Raum.

\section{ZUSAMMENFASSUNG}

1. Es wird eine Glasapparatur zur Bestimmung des Volumens biologischer Objekte beschrieben.

2. Die Genauigkeit der Methode und die Reproduzierbarkeit der Ergebnisse werden an Hand einer Meßreihe dargestellt.

\section{ZITIERTE LITERATUR}

KNIEP, H., 1907. Uber das spezifische Gewicht von Fucus vesiculosus. Ber. dt. bot. Ges. 25, $86-98$.

Kohlrausch, F., 1953. Praktische Physik. Bd. 1. 2. 19. Aufl. 3. Nachdr. Teubner, Leipzig, $544 ; 578 \mathrm{pp}$.

Müller-Stoli, R. W., 1954. Uber Dichte und Sinkvermögen der Thalli bei Großalgen der Ostsee. Flora, Jena 142, 132-145. 